\title{
Lidil
}

Revue de linguistique et de didactique des langues

$45 \mid 2012$

Pratiques de formation à la lecture-écriture des adultes en parcours d'insertion

\section{Balises pour un atelier d'écriture adapté à un public adulte en difficulté}

\section{Maurice Niwese}

\section{(2) OpenEdition}

\section{Journals}

Édition électronique

URL : http://journals.openedition.org/lidil/3199

DOI : 10.4000/lidil.3199

ISSN : 1960-6052

Éditeur

UGA Éditions/Université Grenoble Alpes

Édition imprimée

Date de publication : 15 mai 2012

Pagination : 123-137

ISBN : 978-2-84310-226-4

ISSN : $1146-6480$

\section{Référence électronique}

Maurice Niwese, «Balises pour un atelier d'écriture adapté à un public adulte en diffıculté », Lidil [En ligne], 45 | 2012, mis en ligne le 15 novembre 2013, consulté le 01 mai 2019. URL : http:// journals.openedition.org/lidil/3199; DOI : 10.4000/lidil.3199 


\title{
Balises pour un atelier d'écriture adapté à un public adulte en difficulté
}

\author{
Maurice Niwese*
}

\begin{abstract}
RÉSUMÉ
Rédigé à partir d'une recherche doctorale en didactique de l'écriture, menée dans des centres bruxellois de formation d'adultes en processus d'insertion socioprofessionnelle, cet article analyse d'abord un atelier d'écriture destiné à un public multiculturel en difficulté scripturale et identitaire. Il présente ensuite les fondements théoriques et pédagogiques qui ont présidé à la mise en place d'un nouveau dispositif construit en tenant compte des forces et des limites tant de l'atelier observé que d'autres modèles existants. Il rend enfin compte des principaux résultats obtenus.
\end{abstract}

\begin{abstract}
This paper is based upon doctoral research on the teaching of writing to a group of adults undergoing training for socio-professional integration at some adult education centers in Brussels. It first describes a writing workshop that was intended for a multicultural audience experiencing writing as well as identity difficulties. Then, it presents the theoretical and educational foundations which led to the setting-up of a new model of writing workshops that took into account the strengths and limitations of both the observed writing workshop and other existing models. The paper ends by reporting the main findings of the research.
\end{abstract}

La présente contribution a été rédigée à partir d'une recherche doctorale menée à Bruxelles auprès des publics adultes en formation pour une insertion professionnelle (Niwese, 2010). Cette recherche a été motivée par les difficultés en écriture des populations défavorisées et, à l'opposé, par des recherches en didactique de l'écriture montrant que l'atelier

* Université de Bordeaux 4, IUFM, LACES - E3D; Université catholique de Louvain, CRIPEDIS. 
d'écriture a des effets positifs sur le texte et sur son auteur (LafontTerranova, 2009).

Comme les difficultés en écriture sont un facteur d'exclusion sociale (Artaux, 1999), on peut se demander pourquoi ce dispositif n'est pas utilisé pour les résorber. En cherchant la réponse, on a constaté que la plupart des ateliers portant sur le développement de la compétence scripturale sont adressés à des populations favorisées et que, pour des publics en difficulté, on a tendance à ne viser que la valorisation de soi (Pimet et Boniface, 1999). On a alors voulu savoir si, pour ces derniers, on ne pouvait pas intégrer aussi le travail sur le texte. Ce questionnement a conduit, d'une part, à l'analyse des modèles existants et au suivi d'un atelier d'écriture organisé en milieu défavorisé et, d'autre part, à la construction et à l'expérimentation d'un nouveau dispositif.

Axé sur la phase exploratoire, cet article présente l'atelier d'écriture observé, les fondements théoriques et pédagogiques du nouveau dispositif, et un bref aperçu sur les résultats obtenus.

\section{Forces et limites de l'atelier d'écriture observé}

Le centre qui a accueilli notre enquête exploratoire, Cobs en acronyme anonyme, œuvre dans l'éducation permanente et dans l'insertion socioprofessionnelle. Notre recherche a porté sur un atelier d'écriture, mis en place dans le cadre d'une préformation, dont les visées étaient le développement des compétences en écriture et le renforcement de la confiance en soi.

Au cours de cette phase, nous avons suivi dix stagiaires, âgés de 20 à 38 ans, qui ont fréquenté cet atelier de manière régulière. Issus d'horizons culturels différents (Afrique noire, Maghreb et Occident), infra-scolarisés et sans emploi, ces derniers se posaient des questions identitaires, maitrisaient peu l'écriture et avaient le sentiment de faire partie des exclus de la société. Ils voulaient en revanche améliorer leur rapport à la langue écrite.

L'atelier d'écriture observé s'est tenu en dix séances de trois heures chacune et s'est déroulé entre février et juin 2006. Dans cet atelier, l'activité d'écriture se divisait en quatre moments correspondant au lancement de l'écriture, à la production et à la lecture des textes ainsi qu'aux commentaires. À ces temps s'ajoutait une pratique de réécriture qui permettait à l'animateur de dactylographier certains textes avant de les remettre à leurs auteurs. 
En analysant les sources de l'atelier du Cobs, ses objectifs, ses propositions d'écriture ainsi que la place accordée à l'écriture et à la réécriture, nous allons essayer de cerner ses fondements pédagogiques tout en relevant ses forces et ses limites. Cette analyse sera marquée par l'interaction entre les attentes du public, les visées de l'animateur, nos objectifs de recherche et les connaissances théoriques sur les ateliers d'écriture.

\section{Références aléatoires et visées variables}

L'analyse des déclarations de l'animateur montre que, pour faire écrire, celui-ci s'appuie sur trois principales sources : des ouvrages portant sur les outils et les techniques d'animation d'un atelier d'écriture, les faits divers et le vécu. L'observation des séances a confirmé les déclarations de l'animateur. Ses propositions d'écriture renvoyaient tant aux ateliers centrés sur le texte qu'à ceux qui privilégient le développement personnel. L'animateur s'appuyait aussi sur des apports de cultures différentes, ce qui pouvait être perçu par les participants comme la valorisation et la reconnaissance de la culture de chacun.

Bien que les sources d'écriture aient été diversifiées, leur choix et leur exploitation ont été aléatoires. L'animateur ne programmait pas les faits divers qu'il devait exploiter et il n'avait recours à des ouvrages spécialisés que de façon non planifiée. Quant au vécu, celui-ci semblait convoqué moins pour faire écrire que pour observer le comportement des stagiaires.

L'exploitation aléatoire des références fait penser que l'atelier d'écriture du Cobs reposait moins sur des repères pédagogiques identifiables que sur l'intuition de l'animateur. Cette impression semble confirmée par le déficit d'objectifs clairs.

Comme les objectifs peuvent rester implicites, nous avons, avant et après l'atelier, demandé à l'animateur de nous préciser ses visées. Nous les avons ensuite comparées avec ce qui avait été communiqué aux stagiaires. En analysant les différentes déclarations, on se rend compte que les objectifs varient au fil des jours, certains disparaissant alors que d'autres apparaissent. L'absence d'objectifs clairs n'est pas une difficulté spécifique à l'animateur du Cobs. D'après Boniface, les objectifs de nombre d'animateurs « sont si flous qu'ils seraient bien en peine de les exposer à leurs ouailles » (1992: 15). 


\section{Activités diversifiées dans une logique du fragment}

Les pistes d'écriture proposées au Cobs sont d'une grande variété. On pourrait les répartir sur six axes : le vécu, le vouloir-être, le réel et l'objectif, la fiction, les représentations et l'axe intégrationniste.

L'axe du vécu regrouperait les activités où l'écrit porte sur le sujetécrivant. Les textes à produire sont orientés vers la réalité et portent sur le passé ou sur le présent. L'axe du vouloir-être place aussi le scripteur au cœur de l'écrit, mais, à la différence du premier axe, les textes à produire sont orientés vers le souhait et se rapportent au futur ou au présent. Sur l'axe du réel et de l'objectif, on rangerait les propositions d'écriture qui consistent à faire produire des textes à partir de ce que l'on voit ou de ce que l'on entend. Il s'agit des textes descriptifs ou argumentatifs. L'axe de la fiction regrouperait les activités qui n'ont pas la vocation de décrire la réalité ou d'exprimer le vécu des écrivants. L'axe intégrationniste renverrait aux activités qui font écrire en intégrant des éléments imposés. Enfin, l'axe des représentations rassemblerait les activités portant sur l'image que le scripteur a de l'écriture.

Les activités diversifiées constituent la force de l'atelier observé. En effet, faire écrire en variant les situations d'écriture (entrées et matériaux convoqués) initie les scripteurs à la variété des écrits de la vie pratique (Reuter, 2002). Mais, si on s'arrêtait ici, on risquerait de présenter une réalité tronquée. Les axes décrits ci-dessus sont le fruit de notre propre reconstruction. Dans le concret, les activités s'inscrivaient dans une logique du fragment. Par exemple, pour une durée de trois heures avec une pause de quinze à vingt minutes, certaines séances ont recensé six propositions d'écriture. Cette fragmentation des activités se confirme lorsqu'on considère la place accordée au sujet-écrivant et au processus de la production du texte.

\section{Peu de retour sur le texte produit}

L'atelier du Cobs ne favorise pas le retour sur le texte produit comme cela peut être illustré par le déroulement de trois des quatre moments (la production, la lecture et les commentaires) qui structurent une séance et par la pratique de réécriture en vigueur.

Le temps accordé à la production des textes était court. Comme une seule séance proposait plusieurs activités d'écriture, nombre de stagiaires voyaient venir le temps de présenter leurs textes avant qu'ils aient terminé d'écrire. Les lectures s'enchainaient les unes après les autres sans qu'il y ait un temps pour réagir à la lecture individuelle, alors 
que, si l'on se réfère aux témoignages recueillis, le scripteur souhaitait qu'on l'écoute et qu'on lui parle de son texte. Adressés plutôt au groupe qu'à des écrivants pris particulièrement, les commentaires de l'animateur étaient rarement centrés sur les textes produits.

La pratique de réécriture ne permettait pas non plus au scripteur de retravailler son texte, étant donné que celui-ci était dactylographié par l'animateur. De ce fait, même si l'animateur et les stagiaires sont unanimes pour dire que la dactylographie des textes valorise ces derniers, l'on constate que cette pratique, qui tient le scripteur à l'écart du processus de réécriture, ne lui permet pas de tirer profit des bénéfices didactiques de celle-ci.

Les textes dactylographiés ont été rassemblés dans un livret et envoyés aux stagiaires bien après la fin de la formation. Cet envoi tardif serait lié au fait que le projet de publication des textes a été entrepris vers la fin de l'atelier. Le fait que le recueil n'a pas été remis au groupe dans une des séances de l'atelier a privé celui-ci de l'occasion de valoriser collectivement le travail effectué. De même, comme la socialisation du texte constitue un facteur d'investissement du scripteur, l'animateur aurait pu profiter de ce projet pour faire retravailler les textes par les stagiaires.

\section{Priorité au développement personnel}

Lafont (2009), Rossignol (1996) et Boniface (1992) présentent l'atelier d'écriture du CICLOP (Centre interculturel de communication, langues et orientation pédagogique) comme le représentant emblématique des ateliers d'écriture centrés sur la personne. Même si l'atelier du Cobs ne revendique aucune filiation par rapport aux modèles existants et qu'il met en œuvre des activités relevant de différents courants, il reste proche de celui du CICLOP.

Tant au CICLOP qu'au Cobs, l'atelier vise apparemment la valorisation de soi, le changement du regard sur ses textes et la réconciliation du sujet avec lui-même. De part et d'autre, les activités d'écriture n'entrent pas dans une logique d'ensemble. Chaque séance, voire chaque activité d'écriture, semble se concevoir comme autonome. Au CICLOP comme au Cobs, le temps d'écriture est court, les commentaires font rarement référence à la production textuelle individuelle.

L'orientation de l'atelier du Cobs semble être la même que celle d'autres modèles destinés à des publics en difficulté. Or, la seule valorisation de soi ne répond qu'à une partie des attentes d'un public en difficulté non seulement personnelle, mais aussi scripturale. 


\section{Balises pour un nouveau dispositif}

Construit à partir des forces et des limites des modèles existants, le nouveau dispositif plaide contre une écriture du fragment et pour une cohérence entre les activités mises en œuvre et tient compte de l'hétérogénéité du groupe. Pour cela, il s'inscrit tant dans la pédagogie du projet que dans la pédagogie différenciée, prône une écriture qui va au-delà du premier jet et organise ses activités autour de l'écriture de soi.

\section{Un dispositif inspiré de la pédagogie du projet}

Les données issues tant du terrain observé que des recherches réalisées sur les ateliers d'écriture nous montrent que nombre d'expériences menées déploient une mosaïque d'activités qui n'entrent pas dans la construction d'une logique d'ensemble. Dans nombre de cas, les notions d'objectif, de résultat, de compétence ou d'évaluation sont inconnues. Comme le dispositif élaboré est à visées didactiques, nous avons voulu lutter contre de telles déficiences en l'ancrant dans la pédagogie du projet.

Avant de parler de cette pédagogie, il importe de préciser ce qu'est un projet. La notion de projet trouve son ancrage dans une idéologie qui refuse la prédestination et qui oppose au lendemain inconnu un avenir maitrisé, façonné selon la volonté de l'homme (Tilman, 2004). Les acteurs du projet se fixent des buts, anticipent les résultats, définissent les actions à mener et prévoient les moyens à mobiliser et les mécanismes d'évaluation de ce qui est mis en œuvre. D'après Tilman, pour parler de projet, il faut qu' il y ait intention et réalisation : «Une intention qui ne chercherait pas à se concrétiser ne serait que rêverie; une réalisation qui n'aurait pas été pensée ne serait qu'agitation. » (2004 : 29)

La pédagogie du projet est donc, si l'on peut se permettre d'user de cette tautologie, une pédagogie basée sur le projet. « Tout en évitant les excès analytiques » de la pédagogie par objectifs (Huber, 2005 : 55), cette pédagogie définit les objectifs, fait converger vers eux les activités mises en œuvre et prévoit des mécanismes d'évaluation. Elle vise également « la réalisation d'un produit concret qui fera l'objet d'une socialisation extérieure au groupe formés/formateurs » (Huber, 2005 : 19). Cette socialisation de l'objet réalisé constitue un facteur de motivation, de mobilisation et de valorisation des acteurs.

Pour l'atelier d'écriture expérimenté, nous sommes parti de nos questions de recherche et des attentes du public observé, avons fixé des objectifs et orienté toutes les activités vers leur réalisation. C'est 
aussi par l'inscription dans la pédagogie du projet que nous avons publié les textes produits en dehors du groupe. Cette socialisation étendue a contribué à lutter contre l'écriture du fragment et de l'inachèvement, et poussé les stagiaires à s'investir dans l'écriture et à produire des textes compréhensibles à l'extérieur du lieu de production.

$\mathrm{Si}$, avec la pédagogie du projet, nous avons mis un accent particulier sur le groupe, nous n'avons pas oublié qu'il fallait tenir compte de l'hétérogénéité du public.

\section{Un dispositif inscrit dans la pédagogie différenciée}

La pédagogie différenciée repose sur le postulat qu'à des situations hétérogènes, il faut des réponses hétérogènes. Or, certains pédagogues affirment qu'il n'existe pas de groupe homogène et que ce qui est valable pour un apprenant ne l'est pas forcément pour un autre (Tardif, 2005).

Face à un groupe hétérogène, la différenciation pédagogique peut concerner les contenus, les situations ou les méthodes d'apprentissage. On distingue la différenciation pédagogique successive dite aussi alternative et la différenciation pédagogique simultanée. La première « consiste à faire alterner différentes situations d'apprentissage correspondant aux capacités des apprenants sans modifier fondamentalement le déroulement de la classe et dans le cadre d'une séance commune à tous » (Gillig, 1999 : 60). Pour cette différenciation, la « mise en situation initiale » et les « objectifs poursuivis » restent communs, mais les méthodes et les outils utilisés pour atteindre ces visées sont adaptés « aux capacités de chaque apprenant ». En revanche, pour la différenciation simultanée, les objectifs, les mises en situations initiales, les méthodes et les outils sont différents : "L'idée de simultanéité signifie qu'au même moment les élèves sont occupés à des tâches différentes » (Gillig, 1999 : 61).

Si l'on considère notre dispositif dans son ensemble, on peut dire que nous avons utilisé la différenciation successive. En effet, nous avons visé les mêmes objectifs et nous sommes parti des mêmes activités. Cependant, dans le quotidien de l'expérimentation, la différenciation a été simultanée. Les retards, les absences et la différence de rythmes de travail ont fait qu'au bout de quelques séances, le groupe n'était plus occupé à accomplir les mêmes tâches. Chaque stagiaire a donc suivi son propre chemin et l'accompagnement a été adapté à chacun. 


\section{Un dispositif où l'écriture va au-delà du premier jet}

Les didacticiens de l'écriture misent sur la réécriture pour développer la compétence scripturale des écrivants (Lafont-Terranova, 2009). En effet, en plus d'être inhérente à tout acte d'écriture, la réécriture révèle ce dont le sujet est capable, favorise l'autocorrection et contribue à réduire la surcharge cognitive qui peut être à l'origine de plusieurs imperfections textuelles. Or, dans plusieurs ateliers d'écriture, cette pratique est soit inexistante, soit non systématique, soit accomplie sans l'implication des auteurs des textes concernés.

Lors de l'expérimentation du dispositif, nous avons introduit la réécriture explicitement prescrite de manière progressive. Au départ, la production des textes s'arrêtait au premier jet et, comme au Cobs, nous photocopiions et dactylographiions les textes produits. Ensuite, nous avons fait retravailler les textes en groupe avant de demander aux stagiaires, en troisième et dernière étape, de réécrire eux-mêmes leurs textes. Nous avons retardé la réécriture des textes par leurs auteurs pour permettre à ces derniers de prendre de l'assurance. Les textes personnels ont connu deux grandes réécritures. Ils ont été réécrits d'abord pour être relus par les membres de l'atelier, ensuite pour être publiés en dehors du groupe.

Après la production et la lecture du texte à l'ensemble du groupe, les stagiaires étaient invités à préparer une deuxième version. Celle-ci était destinée à être relue par les pairs ou par le formateur. Cette première réécriture devait permettre de déterminer ce que le stagiaire était capable de faire sans l'intervention manifeste d'un tiers.

Pour amener les stagiaires à encore retravailler leur texte, nous avons mis à leur disposition des cahiers destinés à contenir le manuscrit final qui devait être dactylographié pour être publié. Ce manuscrit correspondait à la version précédente relue et partiellement corrigée par le formateur. Précisément, celui-ci relisait la deuxième version en présence de son auteur, corrigeait une petite partie du texte et suggérait ensuite par des soulignements ce qui pouvait être changé. Cette réécriture accompagnée a donné une idée de ce que le stagiaire changeait avec l'aide manifeste du formateur. Le texte de ce cahier a enfin été corrigé et dactylographié par le formateur avant d'être publié.

Dans le dispositif expérimenté, deux types de textes ont été produits : des textes courts et un texte long dit aussi récit (de soi). C'est ce dernier qui a fait l'objet d'une réécriture systématique. Ce texte a été réalisé en quatre temps. En faisant écrire le récit par morceaux, nous avons voulu montrer aux stagiaires que le récit peut être produit en 
rassemblant des éléments divers dans un tout cohérent. Travailler par morceaux favorise aussi la réécriture, parce que cela requiert des réaménagements constants entre les parties de l'ensemble. Produire un même texte en plusieurs temps et à partir des éléments épars permet par ailleurs de lutter contre des représentations erronées qui veulent qu'il faille avoir tout le texte dans la tête avant de se mettre à écrire (Guibert, 2003).

\section{Un dispositif centré sur la production d'un récit de soi}

Le récit de soi produit dans l'atelier d'écriture expérimenté devait mettre en scène un narrateur-personnage principal qui s'exprime en « je » et qui diffère de l'auteur. Ce récit devait porter sur la famille du personnage, sur son milieu extrafamilial et sur ses perspectives d'avenir. Avant de motiver le choix du récit de soi, explicitons d'abord ce que nous entendons par ce concept.

Lejeune (1996) définit l'autobiographie comme un récit où le personnage central, le narrateur et l'auteur sont identiques. Cette triple identité exclut du champ de l'autobiographie les textes produits dans l'atelier. Cependant, pour nous, de même que nous rejetons, après Ricœur (1985), la fictionnalité pure du récit, de même, nous admettons avec Cyrulnik (2002) que « toute opinion est autobiographique puisqu'elle révèle notre sensibilité au monde ». Mais, pour éviter toute confusion, nous parlons de récit de soi ou de texte narratif de type autobiographique.

\section{Pour une écriture de soi}

Pour (faire) écrire, Reuter identifie quatre sources : «l'imaginaire et la créativité » relevant du patrimoine culturel et de son actualisation par l'imagination, « les savoirs théorico-réflexifs » relatives aux connaissances théoriques sur le texte, « les jeux de la langue et des textes » ainsi que «l'histoire du sujet» (2002: 137-145).

Pour un public comme celui du Cobs, cette dernière source nous a semblé plus appropriée. En effet, de même qu'il nous parait difficile de faire écrire en s'appuyant sur des savoirs théoriques un public qui en est dépourvu, de même, jouer avec la langue pour une personne dont la maitrise linguistique est défaillante pourrait semer la confusion et augmenter les difficultés. Quant au patrimoine culturel, l'hétérogénéité du groupe fait que celui-ci ne partage pas les mêmes références culturelles. En revanche, chacun a une histoire qui lui est accessible et dont la mise en récit peut répondre à des besoins non seulement textuels, mais aussi personnels. 
En effet, comme les stagiaires rencontrés connaissent des parcours de vie chaotiques, le récit de soi peut leur permettre de prendre du recul, d'intellectualiser les évènements, de socialiser l'émotion et de maitriser leurs « affects sans pour autant les nier ou les refouler» (Bucheton, 1995). Le récit de soi permet de se (faire) comprendre dans ce sens que la narration constitue une voie royale conduisant au soi de quelqu'un (Ricœur, 1990) et que le sujet-écrivant met en scène des actions qui le concernent.

\section{Pour contrer les obstacles du récit de soi}

La plaidoirie en faveur de l'écriture de soi ne doit pas faire oublier que cette écriture reste une arme à double tranchant. Dans une situation de formation, elle « comporte des risques [...] de mise en scène des problèmes psychologiques et d'appels dans un espace qui n'est pas de nature thérapeutique » (Reuter, 2002 : 138). D'après le même auteur, pour diminuer les blocages inhérents à l'écriture autobiographique, il faut éviter de solliciter directement le vécu des sujets concernés ou écrire sur des tranches de la vie. Pour nous, l'enjeu a donc été de tirer profit des points positifs de l'écriture de soi et d'éviter son exploitation abusive.

Dans un premier temps, nous avons fait produire des textes courts qui portaient sur des épisodes de la vie des scripteurs tout en précisant qu'il n'y avait aucune obligation d'écrire sur ce qui s'est passé et en insistant sur le fait que tout texte narratif est le fruit de la fiction et de la réalité (Ricœur, 1985).

Dans un second temps, nous avons entamé la production du texte long. Même si les scripteurs étaient avisés qu'il n'était pas question d'écrire une autobiographie comprise au sens de Lejeune (1996), nous les avons entrainés sur le terrain de l'autobiographie en leur demandant de mettre en scène le narrateur-personnage principal s'exprimant en «je ». Cette autobiographie oblique a permis à ceux qui l'ont souhaité de préserver leur intimité en se racontant sous le couvert d'un «je » qui, officiellement, était un autre.

\section{Bref aperçu sur les principaux résultats}

Eu égard au profil du public et à nos questions de recherche, trois objectifs ont été assignés au nouveau dispositif : l'amélioration du rapport à l'écriture, à soi et à autrui, et l'identification des difficultés et des compétences des participants en matière d'écriture. On reprend ici, de façon globale, les résultats obtenus après l'expérimentation. L'analyse a porté 
sur les déclarations orales et écrites des participants ainsi que sur leurs productions textuelles (avant-textes et textes définitifs). Pour ce dernier aspect, on s'est inscrit dans une perspective génétique et linguistique pour étudier l'évolution du volume textuel et les opérations de réécriture, et pour comparer les versions de textes produits avec et sans aide des tiers (pairs et/ou formateur).

\section{L'atelier comme observatoire de l'existant}

La fonction d'observatoire s'avère essentielle quand on a affaire à un public dont on ne peut prévoir a priori ce dont il est capable. L'atelier peut alors être envisagé comme un dispositif qui précède des interventions pédagogiques adaptées aux besoins identifiés.

L'analyse des textes montre que les stagiaires maitrisent plus la macrostructure et la superstructure du récit que sa microstructure. En effet, leurs récits sont narrativement cohérents et recèlent des séquences textuelles variées. En revanche, ils révèlent des défaillances au niveau des microcompétences de la langue écrite.

Sur le plan morphographique, les difficultés semblent liées à l'absence de correspondance univoque entre les graphèmes et les phonèmes. On relève des confusions entre les mots qui, à l'oral, sont homophones ou proches phonétiquement. Sont ainsi confondus à/a, est/es/et/ai, on/ ont, où/ou, ces/ses/ce/se, son/sont/, etc. On observe aussi la difficulté de se représenter les contours morphologiques des unités constitutives de l'énoncé. Dans ce sens, qu'elle donne lieu à quelle, s'est à ces ou à sait, s'était à cette, m'a à ma, m'y à mi, j'habite à jhabite, m'adorait à m'a doré, etc.

La confusion des homophones non homographes ainsi que la difficulté de segmenter les unités du discours seraient des indices du fait que l'oral influence l'écrit des stagiaires et que ceux-ci seraient moins confrontés à l'écrit/ure qu'à l'oral. On peut aussi penser que certaines difficultés (la confusion entre « $\mathrm{i} »$ et $« \mathrm{u} »$, « 1 » et « $\mathrm{r} »$, la nasalisation/ dénasalisation, etc.) seraient liées aux langues d'origine (Niwese, 2010 : 433 et 438). En effet, dans les langues de certains stagiaires, le phonème /y/ et la nasalisation sont inconnus. Chez d'autres, l'opposition entre/r/ et $/ 1 /$ n'est pas pertinente.

L'analyse des marques de référence montre que les défaillances graphiques ont une incidence sur la cohésion textuelle. Ainsi, par exemple, en cas d'homophonie, les reprises «ils », « elles », «sa », «ses » sont, par endroits, rendues par «il », « elle », « ça », « ces». 
Les difficultés liées au « savoir-graphier » n'ont pas empêché les stagiaires de développer des compétences textuelles, grâce notamment à la réécriture. Celle-ci leur a permis d'effectuer des insertions qui relèvent de la présentation, de l'explication, du commentaire, de l'évaluation et de la synthèse. Ils ont aussi réalisé non seulement des modifications formelles, mais aussi des reformulations.

Que les transformations ne se sont pas limitées au niveau de la surface se trouve par ailleurs attesté par des écarts importants entre les volumes textuels d'une version à une autre. L'analyse des textes révèle enfin que, quantitativement, les opérations de réécriture se suivent dans l'ordre décroissant remplacement, ajout, suppression et déplacement $(\mathrm{R}>\mathrm{A}>\mathrm{S}>\mathrm{D})$, ce qui, du point de vue des généticiens du texte, place les stagiaires du côté des scripteurs expérimentés (Fabre-Cols, 2002).

\section{Rapport à soi et à autrui}

À la suite de De Villers (2008), on a défini le rapport à soi et à autrui comme ce que le sujet pense de lui, ce qu'il croit que les autres pensent de lui et ce qu'il pense des autres. D'après les stagiaires, l'atelier a permis un passage de la méfiance à la confiance, et de l'indifférence à la compréhension de l'autre : "Grâce à l'atelier, je me suis sentie proche des autres. De loin, on a souvent une appréhension fausse des autres. Mais en les approchant, [en] conversant avec les différentes personnes, [les] différentes cultures, on comprend mieux la façon de vivre des autres. » (Fiona)

Le regard envers soi a aussi changé. La confrontation au vécu de l'autre a permis à chacun de trouver des points communs avec ses pairs, de relativiser, voire de dépasser ses propres problèmes : «Je vois qu'en écrivant comme ça et en écoutant les gens, je pense qu'on a beaucoup de points communs. Et puis, ça vaut la peine [...] parce que je dis : bon, si j'ai un passé triste [...], je suis pas la seule qui a un passé un peu triste et je peux avancer avec ça.» (Albertine)

Les stagiaires présentent enfin l'atelier comme un lieu où ils se sont sentis écoutés, encouragés et valorisés tant par les pairs que par le formateur.

\section{Rapport à l'écriture}

L'amélioration du rapport à l'écriture (Barré-De Miniac, 2000; Chartrand et Blaser, 2008) a été réalisée dans ses dimensions affective (investissement de l'écriture), axiologique (valeurs, opinions et atti- 
tudes vis-à-vis de l'écriture), conceptuelle (conception de l'écriture et de son apprentissage) et méta-scripturale (verbalisation de sa pratique d'écriture).

D'après les stagiaires, l'atelier d'écriture leur a permis de ne plus avoir peur d'écrire, d'écrire plus facilement qu'auparavant et d'avoir envie de continuer à écrire en dehors de l'atelier. L'analyse de l'évolution du volume textuel et des opérations de réécriture confirme aussi les déclarations des stagiaires. Produire un texte relativement long et le réécrire est, pour quelqu'un qui n'écrit guère, un indice d'investissement dans l'écriture.

Les stagiaires, dont certains se posaient des questions sur l'utilité de l'écriture, ont aussi pris conscience du fait que l'écriture est incontournable dans la société. L'atelier a par ailleurs permis aux participants d'intégrer qu'on apprend à écrire en écrivant, que l'écriture n'est pas une affaire de don et que tout texte est toujours sujet à des modifications. Enfin, à la fin de l'atelier, les stagiaires étaient capables de parler de l'écriture en général et de leurs pratiques scripturales en particulier.

\section{Conclusion}

Comme c'est le cas pour nombre d'autres modèles d'ateliers existants, l'atelier du Cobs met en place une mosaïque d'activités qui ne construisent pas un ensemble conçu comme une unité cohérente. Cet atelier vise aussi le développement personnel, ce qui le rapproche des modèles que l'on propose à des publics en difficulté. Or, cette orientation ne répond qu'à une partie des attentes du public rencontré.

Le dispositif élaboré a été inscrit dans la pédagogie du projet pour lutter contre la logique du fragment et dans la pédagogie différenciée pour tenir compte de l'hétérogénéité du groupe. Ce dispositif accorde par ailleurs une place privilégiée à la réécriture et rompt ainsi avec une certaine tradition qui, face à un public en situation difficile, ne vise que la valorisation de soi. Il a enfin été centré sur la production du récit de soi pour travailler des questions textuelles et personnelles.

Le bref aperçu fait sur les résultats obtenus montre que ce nouveau dispositif est un outil de diagnostic de l'existant et qu'il permet aux participants d'améliorer leur rapport à eux-mêmes, aux autres et à l'écriture. Les résultats révèlent aussi que les stagiaires ont été animés par une réelle volonté de mieux écrire, mais que, chemin faisant, ils ont été confrontés à des difficultés relevant surtout du savoir-graphier. Pour un public comme celui qui a fait l'objet de notre expérience, le grand défi 
serait d'arriver à acquérir des microcompétences de la langue écrite, qui n'ont pas été suffisamment intégrées, suite à des raisons variées ayant surtout trait au parcours personnel de chacun.

\section{RÉFÉRENCES BIBLIOGRAPHIQUES}

Artaux M.-F. (1999) : Entre l'enfant et l'élève, l'écriture de soi. Produire, cheminer, penser, exister dans un atelier d'écriture, Nancy, Presses universitaires de Nancy.

BARRÉ-De Miniac C. (2000) : Le rapport à l'écriture, Villeneuve-d'Ascq, Presses universitaires du Septentrion.

Boniface C. (1992): Les ateliers d'écriture, Paris, Retz.

Bucheton D. (1995) : Écriture Réécritures. Récits d'adolescents, Berne, Peter Lang.

Chartrand S.-G. et Blaser C. (2008) : « Du rapport à l'écriture au concept didactique de capacités langagières. Apports et limites de la notion de rapport à l'écrit », dans S.-G. Chartrand et C. Blaser (dir.), Le rapport à l'écrit. Un outil pour enseigner de l'école à l'université, Namur, Presses universitaires de Namur, p. 107-127.

Cyrulnik B. (2002) : Un merveilleux malheur, Paris, Odile Jacob.

Fabre-Cols C. (2002) : Réécrire à l'école et au collège. De l'analyse des brouillons à l'écriture accompagnée, France, ESF.

GiLlig J.-M. (dir.) (1999) : Les pédagogies différenciées. Origine, actualité et perspectives, Paris-Bruxelles, De Boeck Université.

GUIBERT R. (2003) : Former des écrivants, Villeneuve-d'Ascq, Presses universitaires du Septentrion.

Huber M. (2005) : Apprendre en projets. La pédagogie du projet-élèves, Lyon, Chronique sociale.

LAFONT-TERRANOVA J. (2009) : Se construire, à l'école, comme sujet-écrivant. L'apport des ateliers d'écriture, Namur, Presses universitaires de Namur.

Lejeune P. (1996) : Le Pacte autobiographique, Paris, Seuil.

Niwese M. (2010) : L'atelier d'écriture. Un dispositif didactique pour apprendre à écrire à un groupe multiculturel d'adultes en reprise de formation, Louvain-la-Neuve, université catholique de Louvain.

Pimet O. et Boniface C. (1999) : Ateliers d'écriture. Mode d'emploi, Paris, ESF.

REUTER Y. (2002) : Enseigner et apprendre à écrire, Paris, ESF.

Rossignol I. (1996) : L'invention des ateliers d'écriture en France. Analyse comparative de sept courants clés, Paris, L'Harmattan.

Riceur P. (1985): Temps et récit. 3. Le temps raconté, Paris, Seuil.

Riceur P. (1990) : Soi-même comme un autre, Paris, Seuil. 
TARDIF N. (2005) : «La pédagogie différenciée au service de l'apprentissage », Vie pédagogique, n 134, p. 21-24.

Tilman F. (2004) : Penser le projet. Concepts et outils d'une pédagogie émancipatrice, Lyon, Chronique sociale.

VILLERS G. (DE) (2008) : «Identité versus subjectivité dans le récit autobiographique et en formation d'adultes », Le Journal de l'Alpha, $\mathrm{n}^{\circ} 166$, p. $35-40$. 
